\title{
Strong Convergence of Iterative Algorithm for a New System of Generalized $H(\cdot, \cdot)-\eta$-Cocoercive Operator Inclusions in Banach Spaces
}

\author{
Saud M. Alsulami, ${ }^{1}$ Eskandar Naraghirad, ${ }^{2}$ and Nawab Hussain ${ }^{1}$ \\ ${ }^{1}$ Department of Mathematics, King Abdulaziz University, P.O. Box 80203, Jeddah 21589, Saudi Arabia \\ ${ }^{2}$ Department of Mathematics, Yasouj University, Yasouj 75918, Iran \\ Correspondence should be addressed to Saud M. Alsulami; alsulami@kau.edu.sa
}

Received 10 September 2013; Revised 6 November 2013; Accepted 20 November 2013

Academic Editor: Mohammad Mursaleen

Copyright ( $) 2013$ Saud M. Alsulami et al. This is an open access article distributed under the Creative Commons Attribution License, which permits unrestricted use, distribution, and reproduction in any medium, provided the original work is properly cited.

\begin{abstract}
We introduce and study a new system of generalized $H(\cdot, \cdot)-\eta$-cocoercive operator inclusions in Banach spaces. Using the resolvent operator technique associated with $H(\cdot, \cdot)-\eta$-cocoercive operators, we suggest and analyze a new generalized algorithm of nonlinear set-valued variational inclusions and establish strong convergence of iterative sequences produced by the method. We highlight the applicability of our results by examples in function spaces.
\end{abstract}

\section{Introduction}

The resolvent operator technique is a powerful tool to study the approximation solvability of nonlinear variational inequalities and variational inclusions, which have been applied widely to optimization and control, mechanics and physics, economics and transportation equilibrium, and engineering sciences, see, for example, $[1-4]$ and the references therein.

In a series of papers [5-8], the authors investigated $(A, \eta)$-accretive and $H(\cdot, \cdot)$-accretive operators for solving variational inclusions in Banach spaces. Convergence and stability of iterative algorithms for the systems of $(A, \eta)$ accretive operators have been studied in $[9,10]$. The notion of $(H, \phi)-\eta$-monotone operators has been introduced and investigated by the authors in [11]. Generalized mixed variational inclusions involving $(H(\cdot, \cdot), \eta)$-monotone operators have been discussed in [12]. Some results on $H((\cdot, \cdot), \eta)$ accretive operators and application for solving set-valued variational inclusions in Banach spaces have been proved in [7]. Some other related articles on the variational inclusion problems can be found in [13-22].
Very recently, Ahmad et al. [23] introduced a new $H(\cdot, \cdot)-$ $\eta$-cocoercive operator and its resolvent operator in the setting of Banach spaces. The authors proposed concrete examples in support of $H(\cdot, \cdot)-\eta$-cocoercive operators and they also proved the Lipschitz continuity of resolvent operator associated with $H(\cdot, \cdot)-\eta$-cocoercive operator. Motivated and inspired by the research works mentioned above, in this paper, we introduce and study a new system of $H(\cdot, \cdot)$ $\eta$-cocoercive mapping inclusions in Banach spaces. Using the resolvent operator associated with $H(\cdot, \cdot)-\eta$-cocoercive mapping, we suggest and analyze a new general algorithm and establish the existence and uniqueness of solutions for this system of $H(\cdot, \cdot)-\eta$-cocoercive mappings.

\section{Preliminaries}

Throughout this paper, we denote the set of positive integers by $\mathbb{N}$. Let $X$ be a Banach space with the norm $\|\cdot\|$ and the dual space $X^{*}$. For any $x \in X$, we denote the value of $x^{*} \in X^{*}$ at $x$ by $\left\langle x, x^{*}\right\rangle$. When $\left\{x_{n}\right\}$ is a sequence in $X$, we denote the strong convergence of $\left\{x_{n}\right\}$ to $x \in X$ by $x_{n} \rightarrow x$ as $n \rightarrow \infty$. We denote by $2^{X}$ the family of all nonempty subsets of $X$. Let 
$C B(X)$ be the family of all nonempty, closed, and bounded subsets of $X$. The Hausdörff metric on $C B(X)[24]$ is defined by

$$
\begin{array}{r}
D(A, B)=\max \left\{\sup _{x \in A} d(x, B), \sup _{y \in B} d(A, y)\right\}, \\
A, B \in C B(X),
\end{array}
$$

where $d(x, B)=\inf _{b \in B}\|x-b\|$ and $d(A, y)=\inf _{a \in A}\|a-y\|$.

Definition 1 (see [25]). A continuous and strictly increasing function $\phi:[0,+\infty) \rightarrow[0, \infty)$ such that $\phi(0)=0$ and $\lim _{t \rightarrow \infty} \phi(t)=\infty$ is called a gauge function.

Definition 2 (see [25]). Let $X$ be a Banach space. Given a gauge function $\phi$, the mapping $J_{\phi}: X \rightarrow 2^{X^{*}}$ corresponding to $\phi$ defined by

$$
\begin{array}{r}
J_{\phi}(x)=\left\{x^{*} \in X^{*}:\left\langle x, x^{*}\right\rangle=\|x\|\left\|x^{*}\right\|,\right. \\
\left.\left\|x^{*}\right\|=\phi(\|x\|)\right\}, \quad \forall x \in X,
\end{array}
$$

is called the duality mapping with gauge function $\phi$.

In particular, if $\phi(t)=t$, the duality map $J=J_{\phi}$ is called the normalized duality mapping.

Lemma 3 (see [26]). Let $X$ be a real Banach space and $J$ : $X \rightarrow 2^{X^{*}}$ be the normalized duality mapping. Then, for any $x, y \in X$,

$$
\|x+y\|^{2} \leq\|x\|^{2}+2\langle y, j(x+y)\rangle,
$$

for all $j(x+y) \in J(x+y)$.

Definition 4. Let $X$ be a Banach space. Let $A: X \rightarrow X$ and $\eta: X \times X \rightarrow X$ be two mappings and $J: X \rightarrow 2^{X^{*}}$ be the normalized duality mapping. Then, $A$ is called

(i) $\eta$-cocoercive, if there exists a constant $\mu_{1}>0$ such that

$$
\begin{array}{r}
\langle A x-A y, j(\eta(x, y))\rangle \geq \mu_{1}\|A x-A y\|^{2}, \quad \forall x, y \in X, \\
j(\eta(x, y)) \in J(\eta(x, y)),
\end{array}
$$

(ii) $\eta$-accretive, if

$$
\begin{array}{r}
\langle A x-A y, j(\eta(x, y))\rangle \geq 0, \quad \forall x, y \in X, \\
j(\eta(x, y)) \in J(\eta(x, y)),
\end{array}
$$

(iii) $\eta$-strongly accretive, if there exists a constant $\beta_{1}>0$ such that

$$
\begin{array}{r}
\langle A x-A y, j(\eta(x, y))\rangle \geq \beta_{1}\|x-y\|^{2}, \quad \forall x, y \in X, \\
j(\eta(x, y)) \in J(\eta(x, y)),
\end{array}
$$

(iv) $\eta$-relaxed cocoercive, if there exists a constant $\gamma_{1}>0$ such that

$$
\begin{array}{r}
\langle A x-A y, j(\eta(x, y))\rangle \geq\left(-\gamma_{1}\right)\|A x-A y\|^{2}, \quad \forall x, y \in X, \\
j(\eta(x, y)) \in J(\eta(x, y)),
\end{array}
$$

(v) Lipschitz continuous, if there exists a constant $\lambda_{A}>0$ such that

$$
\|A x-A y\| \leq \lambda_{A}\|x-y\|, \quad \forall x, y \in X,
$$

(vi) $\alpha$-expansive, if there exists a constant $\alpha>0$ such that

$$
\|A x-A y\| \geq \alpha\|x-y\|, \quad \forall x, y \in X,
$$

(vii) $\eta$ is said to be Lipschitz continuous, if there exists a constant $\tau>0$ such that

$$
\|\eta(x, y)\| \leq \tau\|x-y\|, \quad \forall x, y \in X .
$$

Definition 5. Let $X$ be a Banach space. Let $A, B: X \rightarrow X, H$ : $X \times X \rightarrow X, \eta: X \times X \rightarrow X$ be four single-valued mappings and $J: X \rightarrow 2^{X^{*}}$ be the normalized duality mapping. Then,

(i) $H(A, \cdot)$ is said to be $\eta$-cocoercive with respect to $A$, if there exists a constant $\mu>0$ such that

$$
\begin{gathered}
\langle H(A x, u)-H(A y, u), j(\eta(x, y))\rangle \\
\geq \mu\|A x-A y\|^{2}, \quad \forall x, y, u \in X, \\
j(\eta(x, y)) \in J(\eta(x, y)),
\end{gathered}
$$

(ii) $H(\cdot, B)$ is said to be $\eta$-relaxed cocoercive with respect to $B$, if there exists a constant $\gamma>0$ such that

$$
\begin{array}{r}
\langle H(u, B x)-H(u, B y), j(\eta(x, y))\rangle \\
\geq(-\gamma)\|B x-B y\|^{2}, \quad \forall x, y, u \in X, \\
j(\eta(x, y)) \in J(\eta(x, y)),
\end{array}
$$

(iii) $H(A, \cdot)$ is said to be $r_{1}$-Lipschitz continuous with respect to $A$, if there exists a constant $r_{1}>0$ such that

$\|H(A x, u)-H(A y, u)\| \leq r_{1}\|x-y\|, \quad \forall x, y, u \in X$,

(iv) $H(\cdot, B)$ is said to be $r_{2}$-Lipschitz continuous with respect to $B$, if there exists a constant $r_{2}>0$ such that

$$
\|H(u, B x)-H(u, B y)\| \leq r_{2}\|x-y\|, \quad \forall x, y, u \in X .
$$

Definition 6. Let $X$ be a Banach space. A set-valued mapping $M: X \rightarrow 2^{X}$ is said to be $\eta$-cocoercive, if there exists a constant $\mu_{2}>0$ such that

$$
\begin{aligned}
& \langle u-v, j(\eta(x, y))\rangle \geq \mu_{2}\|u-v\|^{2}, \quad \forall x, y \in X, \\
& u \in M(x), \quad v \in M(y), \quad j(\eta(x, y)) \in J(\eta(x, y)) .
\end{aligned}
$$

Definition 7. Let $X$ be a Banach space. A mapping $T: X \rightarrow$ $C B(X)$ is said to be $\mathscr{D}$-Lipschitz continuous, if there exists a constant $\lambda_{T}>0$ such that

$$
\mathscr{D}(T x, T y) \leq \lambda_{T}\|x-y\|, \quad x, y \in X .
$$


Definition 8. Let $X$ be a Banach space. Let $T, Q: X \rightarrow$ $C B(X)$ be the mappings. A mapping $N: X \times X \rightarrow X$ is said to be

(i) Lipschitz continuous in the first argument with respect to $T$, if there exists a constant $t_{1}>0$ such that

$$
\begin{array}{r}
\left\|N\left(w_{1}, \cdot\right)-N\left(w_{2}, \cdot\right)\right\| \leq t_{1}\left\|w_{1}-w_{2}\right\|, \quad \forall u_{1}, u_{2} \in X, \\
w_{1} \in T\left(u_{1}\right), \quad w_{2} \in T\left(u_{2}\right),
\end{array}
$$

(ii) Lipschitz continuous in the second argument with respect to $Q$, if there exists a constant $t_{2}>0$ such that

$$
\begin{array}{r}
\left\|N\left(\cdot, v_{1}\right)-N\left(\cdot, v_{2}\right)\right\| \leq t_{2}\left\|v_{1}-v_{2}\right\|, \quad \forall u_{1}, u_{2} \in X, \\
v_{1} \in Q\left(u_{1}\right), \quad v_{2} \in Q\left(u_{2}\right),
\end{array}
$$

(iii) $\eta$-relaxed Lipschitz in the first argument with respect to $T$, if there exists a constant $\tau_{1}>0$ such that

$$
\begin{array}{r}
\left\langle N\left(w_{1}, \cdot\right)-N\left(w_{2}, \cdot\right), j\left(\eta\left(u_{1}, u_{2}\right)\right)\right\rangle \\
\leq\left(-\tau_{1}\right)\left\|u_{1}-u_{2}\right\|^{2}, \quad \forall u_{1}, u_{2} \in X, \\
w_{1} \in T\left(u_{1}\right), \quad w_{2} \in T\left(u_{2}\right), \\
j\left(\eta\left(u_{1}, u_{2}\right)\right) \in J\left(\eta\left(u_{1}, u_{2}\right)\right),
\end{array}
$$

(iv) $\eta$-relaxed Lipschitz in the second argument with respect to $Q$, if there exists a constant $\tau_{2}>0$ such that

$$
\begin{aligned}
& \left\langle N\left(\cdot, v_{1}\right)-N\left(\cdot, v_{2}\right), j\left(\eta\left(u_{1}, u_{2}\right)\right)\right\rangle \\
& \leq\left(-\tau_{2}\right)\left\|u_{1}-u_{2}\right\|^{2}, \quad \forall u_{1}, u_{2} \in X, \quad v_{1} \in Q\left(u_{1}\right), \\
& \quad v_{2} \in Q\left(u_{2}\right), \quad j\left(\eta\left(u_{1}, u_{2}\right)\right) \in J\left(\eta\left(u_{1}, u_{2}\right)\right) .
\end{aligned}
$$

Definition 9. Let $X$ be a Banach space. Let $A, B: X \rightarrow X$, $H: X \times X \rightarrow X, \eta: X \times X \rightarrow X$ be four single-valued mappings. Let $M: X \rightarrow 2^{X}$ be a set-valued mapping. $M$ is said to be $H(\cdot, \cdot)-\eta$-cocoercive operator with respect to $A$ and $B$, if $M$ is $\eta$-cocoercive and $(H(A, B)+\lambda M)(X)=X$, for every $\lambda>0$.

Example 10. Let $X=\mathbb{R} \times \mathbb{R}$ and $A, B: X \rightarrow X$ be defined by

$$
\begin{aligned}
& A\left(x_{1}, x_{2}\right)=\left(2 x_{1}-x_{2}, x_{1}-x_{2}\right), \\
& B\left(y_{1}, y_{2}\right)=\left(-2 y_{2}, y_{1}-y_{2}\right), \quad \forall\left(x_{1}, x_{2}\right),\left(y_{1}, y_{2}\right) \in X .
\end{aligned}
$$

Assume now that $H(A, B), \eta: X \times X \rightarrow X$ are defined by

$$
H(A x, B y)=A x+B y, \quad \eta(x, y)=x-y, \quad \forall x, y \in X .
$$

Let $M=I$, where $I$ is the identity mapping. Then, $M$ is $H(\cdot, \cdot)-\eta$-cocoercive with respect to $A$ and $B$.
Example 11. Let $X=C[0,1]$, the space of all real valued continuous functions defined on closed interval $[0,1]$ with the norm

$$
\|f\|=\max _{t \in[0,1]}|f(t)| .
$$

Let $A, B: X \rightarrow X$ be defined by

$$
A(f)=\sin ^{2}(f), \quad B(g)=\cos ^{2}(g), \quad \forall f, g \in X .
$$

Let $H(A, B): X \times X \rightarrow X$ be defined by

$$
H(A(f), B(g))=A(f)+B(g), \quad \forall f, g \in X .
$$

Suppose that $M(f)=f^{2}$, where $f^{2}(t)=f(t) f(t)$ for all $t \in$ $[0,1]$. Then, for $\lambda=1$, we conclude that

$$
\begin{aligned}
& \|(H(A, B)+M)(f)\|=\left\|A(f)+B(f)+f^{2}\right\| \\
& \quad=\max _{t \in[0,1]}\left|\sin ^{2}(f(t))+\cos ^{2}(f(t))+f^{2}(t)\right| \\
& \quad=1+f^{2}(t)>0 .
\end{aligned}
$$

This proves that $0 \notin(H(A, B)+M)(X)$ and $M$ is not $H(A, B)-$ $\eta$-cocoercive with respect to $A$ and $B$.

Proposition 12 (see [23]). Let $H(A, B)$ be $\eta$-cocoercive with respect to $A$ with constant $\mu>0$ and $\eta$-relaxed cocoercive with respect to $B$ with constant $\gamma>0, A$ be $\alpha$-expansive and $B$ be $\beta$-Lipschitz continuous $\mu>\gamma$ and $\alpha>\beta$. Let $M: X \rightarrow 2^{X}$ be $H(A, B)-\eta$-cocoercive operator. Suppose that

$$
\begin{array}{r}
\langle x-y, j(\eta(u, v))\rangle \geq 0, \quad \forall(v, y) \in \operatorname{Graph}(M), \\
j(\eta(u, v)) \in J(\eta(u, v)) .
\end{array}
$$

Then, $x \in M u$, where $G r a p h(M)=\{(u, x) \in X \times X: x \in M u\}$.

Theorem 13 (see [23]). Let $H(A, B)$ be $\eta$-cocoercive with respect to $A$ with constant $\mu>0$ and $\eta$-relaxed cocoercive with respect to $B$ with constant $\gamma>0, A$ be $\alpha$-expansive and $B$ be $\beta$-Lipschitz continuous, $\mu>\gamma$ and $\alpha>\beta$. Let $M$ be an $H(\cdot, \cdot)-\eta$-cocoercive operator with respect to $A$ and $B$. Then, for each $\lambda>0$, the operator $(H(A, B)+\lambda M)^{-1}$ is single-valued.

Definition 14. Let $X$ be a Banach space. Let $H(A, B)$ be $\eta$ cocoercive with respect to $A$ with constant $\mu>0$ and $\eta$ relaxed cocoercive with respect to $B$ with constant $\gamma>0$, $A$ be $\alpha$-expansive $B$ be $\beta$-Lipschitz continuous and $\eta$ be $\beta$ Lipschitz continuous, $\mu>\gamma$, and $\alpha>\beta$. Let $M$ be a $H(\cdot, \cdot)-\eta$-cocoercive operator with respect to $A$ and $B$. Then, the resolvent $R_{\lambda, M}^{H(\cdot,)-\eta}: X \rightarrow X$ is defined by

$$
R_{\lambda, M}^{H(\cdot, \cdot)-\eta}(u)=(H(A, B)+\lambda M)^{-1}(u), \quad \forall u \in X .
$$

Theorem 15 (see [23]). Let $X$ be a Banach space. Let $H(A, B)$ be $\eta$-cocoercive with respect to $A$ with constant $\mu>0$ and $\eta$ relaxed cocoercive with respect to $B$ with constant $\gamma>0, A$ be $\alpha$-expansive $B$ be $\beta$-Lipschitz continuous, and $\eta$ be $\rho$-Lipschitz continuous; $\mu>\gamma$ and $\alpha>\beta$. Let $M$ be $H(\cdot, \cdot)-\eta$-cocoercive 
operator with respect to $A$ and $B$. Then, the resolvent operator $R_{\lambda, M}^{H(\cdot, \cdot)-\eta}: X \rightarrow X$ is $\rho /\left(\mu \alpha^{2}-\gamma \beta^{2}\right)$-Lipschitz continuous, that is,

$$
\begin{aligned}
& \left\|R_{\lambda, M}^{H(\cdot,)-\eta}(u)-R_{\lambda, M}^{H(\cdot, \cdot)-\eta}(v)\right\| \\
& \quad \leq \frac{\rho}{\mu \alpha^{2}-\gamma \beta^{2}}\|u-v\|, \quad \forall u, v \in X .
\end{aligned}
$$

\section{Strong Convergence Theorem}

In this section, using the resolvent operator technique associated with $H(\cdot, \cdot)-\eta$-cocoercive operators, we propose a new generalized algorithm of nonlinear set-valued variational inclusions and establish strong convergence of iterative sequences produced by the method.

For $i=1,2$, let $X_{i}$ be real Banach spaces with the norm $\|\cdot\|_{i}$. Let $A_{i}, B_{i}: X_{i} \rightarrow X_{i}, H_{i}: X_{i} \times X_{i} \rightarrow X_{i}, \eta_{i}: X_{i} \times$ $X_{i} \rightarrow X_{i}, F: X_{1} \times X_{2} \rightarrow X_{1}$, and $G: X_{1} \times X_{2} \rightarrow X_{2}$ be single-valued mappings, and $T: X_{1} \rightarrow C B\left(X_{1}\right), Q: X_{2} \rightarrow$ $C B\left(X_{2}\right)$ be set-valued mappings. Let $M: X_{1} \times X_{1} \rightarrow 2^{X_{1}}$, $N: X_{2} \times X_{2} \rightarrow 2^{X_{2}}$ be $H_{1}(\cdot, \cdot)-\eta_{1}$-cocoercive and $H_{2}(\cdot, \cdot)-$ $\eta_{2}$-cocoercive operators with respect to $\left(A_{1}, B_{1}\right)$ and $\left(A_{2}, B_{2}\right)$, respectively. We consider the following problem.

Find $(x, y) \in X_{1} \times X_{2}, w \in T(x)$, and $v \in Q(y)$ such that

$$
\begin{aligned}
& 0 \in M(x, x)+F(w, v), \\
& 0 \in N(y, y)+G(w, v) .
\end{aligned}
$$

We call problem (30) a system of generalized $H(\cdot, \cdot)-\eta$ cocoercive operator inclusions.

Under the assumptions mentioned above, we have the following key and simple lemma.

Lemma 16. $(x, y) \in X_{1} \times X_{2}, w \in T(x), v \in Q(y)$ is a solution of problem (30) if and only if

$$
\begin{aligned}
& x=R_{\lambda_{1}, M(\cdot, x)}^{H_{1}(\cdot,)-\eta}\left[H_{1}\left(A_{1} x, B_{1} x\right)-\lambda_{1} F(w, v)\right], \\
& y=R_{\lambda_{2}, N(\cdot, y)}^{H_{2}(\cdot, \cdot)-\eta}\left[H_{2}\left(A_{2} y, B_{2} y\right)-\lambda_{2} G(w, v)\right],
\end{aligned}
$$

where $R_{\lambda_{1}, M(\cdot, x)}^{H_{1}(\cdot, \cdot)-\eta}=\left(H_{1}\left(A_{1} x, B_{1} x\right)+\lambda_{1} M(\cdot, x)\right)^{-1}, R_{\lambda_{1}, N(\cdot, y)}^{H_{2}(\cdot, \cdot)-\eta}=$ $\left(H_{2}\left(A_{2} y, B_{2} y\right)+\lambda_{2} N(\cdot, y)\right)^{-1}$, and $\lambda_{1}, \lambda_{2}>0$ are constants.

Proof. This is an easy and direct consequence of Definition 14.

Algorithm 17. For $i=1,2$, let $X_{i}$ be real Banach spaces with the norm $\|\cdot\|_{i}$. Let $A_{i}, B_{i}: X_{i} \rightarrow X_{i}, H_{i}: X_{i} \times X_{i} \rightarrow X_{i}$, $\eta_{i}: X_{i} \times X_{i} \rightarrow X_{i}, F: X_{1} \times X_{2} \rightarrow X_{1}$, and $G: X_{1} \times X_{2} \rightarrow X_{2}$ be single-valued mappings, and $T: X_{1} \rightarrow C B\left(X_{1}\right), Q$ : $X_{2} \rightarrow C B\left(X_{2}\right)$ be set-valued mappings. Let $M: X_{1} \times X_{1} \rightarrow$ $\rightarrow 2^{X_{1}}, N: X_{2} \times X_{2} \rightarrow 2^{X_{2}}$ be such that, for each fixed $x \in X_{1}, y \in X_{2}, M(\cdot, x)$ and $N(\cdot, y)$ are $H_{1}(\cdot, \cdot)-\eta_{1}$-cocoercive and $H_{2}(\cdot, \cdot)-\eta_{2}$-cocoercive operators with respect to $\left(A_{1}, B_{1}\right)$ and $\left(A_{2}, B_{2}\right)$, respectively. For any given constants $\lambda_{i}>0$ $(i=1,2)$, define the mappings $S_{1}: X_{1} \times X_{2} \rightarrow X_{1}$ and $S_{2}: X_{1} \times X_{2} \rightarrow X_{2}$ by

$$
\begin{aligned}
& S_{1}(x, y) \\
& \quad=\bigcup_{w \in T(x)} \bigcup_{v \in Q(y)} R_{\lambda_{1}, M(\cdot, x)}^{H_{1}(\cdot, \cdot)-\eta_{1}}\left[H_{1}\left(A_{1} x, B_{1} x\right)-\lambda_{1} F(w, v)\right], \\
& S_{2}(x, y) \\
& \quad=\bigcup_{w \in T(x)} \bigcup_{v \in Q(y)} R_{\lambda_{2}, N(\cdot, y)}^{H_{2}(\cdot, \cdot)-\eta_{2}}\left[H_{2}\left(A_{2} y, B_{2} y\right)-\lambda_{2} G(w, v)\right] .
\end{aligned}
$$

For any given $\left(x_{0}, y_{0}\right) \in X_{1} \times X_{2}, w_{0} \in T\left(x_{0}\right), v_{0} \in Q\left(y_{0}\right)$, let

$$
\begin{aligned}
z_{0}= & R_{\lambda_{1}, M\left(\cdot, x_{0}\right)}^{H_{1}(\cdot,)-\eta_{1}} \\
& \times\left[H_{1}\left(A_{1} x_{0}, B_{1} x_{0}\right)-\lambda_{1} F\left(w_{0}, v_{0}\right)\right] \in S_{1}\left(x_{0}, y_{0}\right), \\
u_{0}= & R_{\lambda_{2}, N\left(\cdot, y_{0}\right)}^{H_{2}(\cdot,)-\eta_{2}} \\
& \times\left[H_{2}\left(A_{2} y_{0}, B_{2} y_{0}\right)-\lambda_{2} G\left(w_{0}, v_{0}\right)\right] \in S_{2}\left(x_{0}, y_{0}\right) .
\end{aligned}
$$

Since $w_{0} \in T\left(x_{0}\right) \subset C B\left(X_{1}\right)$ and $v_{0} \in Q\left(y_{0}\right) \subset C B\left(X_{2}\right)$, in view of Nadler's theorem [24], there exist $w_{1} \in T\left(x_{1}\right)$ and $v_{1} \in Q\left(y_{1}\right)$ such that

$$
\begin{aligned}
& \left\|w_{1}-w_{0}\right\|_{1} \leq(1+1) D\left(T\left(x_{1}\right), T\left(x_{0}\right)\right), \\
& \left\|v_{1}-v_{0}\right\|_{2} \leq(1+1) D\left(Q\left(y_{1}\right), Q\left(y_{0}\right)\right) .
\end{aligned}
$$

By induction, we define iterative sequences $\left\{x_{n}\right\},\left\{y_{n}\right\},\left\{w_{n}\right\}$, and $\left\{v_{n}\right\}$ as follows:

$$
\begin{aligned}
x_{n+1}= & R_{\lambda_{1}, M\left(\cdot, x_{n}\right)}^{H_{1}(\cdot \cdot)-\eta_{1}} \\
& \times\left[H_{1}\left(A_{1} x_{n}, B_{1} x_{n}\right)-\lambda_{1} F\left(w_{n}, v_{n}\right)\right] \in S_{1}\left(x_{n}, y_{n}\right), \\
y_{n+1}= & R_{\lambda_{2}, N\left(\cdot, y_{n}\right)}^{H_{2}(\cdot,)-\eta_{2}} \\
& \times\left[H_{2}\left(A_{2} y_{n}, B_{2} y_{n}\right)-\lambda_{2} G\left(w_{n}, v_{n}\right)\right] \in S_{2}\left(x_{n}, y_{n}\right),
\end{aligned}
$$

$w_{n} \in T\left(x_{n}\right)$,

$\left\|w_{n+1}-w_{n}\right\|_{1} \leq\left(1+\frac{1}{n+1}\right) D\left(T\left(x_{n+1}\right), T\left(x_{n}\right)\right)$,

$v_{n} \in Q\left(y_{n}\right)$,

$\left\|v_{n+1}-v_{n}\right\|_{2} \leq\left(1+\frac{1}{n+1}\right) D\left(Q\left(y_{n+1}\right), Q\left(y_{n}\right)\right)$,

where $n=0,1,2, \ldots$, and $\lambda_{1}, \lambda_{2}>0$ are constants.

Theorem 18. For $i=1,2$, let $X_{i}$ be real Banach spaces with the norm $\|\cdot\|_{i}$. Let $A_{i}, B_{i}: X_{i} \rightarrow X_{i}, H_{i}: X_{i} \times X_{i} \rightarrow X_{i}$, $\eta_{i}: X_{i} \times X_{i} \rightarrow X_{i}, F: X_{1} \times X_{2} \rightarrow X_{1}$ and $G: X_{1} \times X_{2} \rightarrow X_{2}$ be single-valued mappings, and $T: X_{1} \rightarrow C B\left(X_{1}\right), Q: X_{2} \rightarrow$ 
$C B\left(X_{2}\right)$ be set-valued mappings. Let $M: X_{1} \times X_{1} \rightarrow 2^{X_{1}}$ and $N: X_{2} \times X_{2} \rightarrow 2^{X_{2}}$ be such that, for each fixed $x \in$ $X_{1}, y \in X_{2}, M(\cdot, x)$ and $N(\cdot, y)$ are $H_{1}(\cdot, \cdot)-\eta_{1}$-cocoercive and $H_{2}(\cdot, \cdot)-\eta_{2}$-cocoercive operators with respect to $\left(A_{1}, B_{1}\right)$ and $\left(A_{2}, B_{2}\right)$, respectively. Suppose that the following conditions are satisfied.

(i) $H_{i}\left(A_{i}, B_{i}\right)$ is $\eta_{i}$-cocoercive with respect to $A_{i}$ with constant $\mu_{i}$ and $\eta_{i}$-relaxed cocoercive with respect to $B_{i}$ with constant $\gamma_{i}, i=1,2$.

(ii) $A_{i}$ is $\alpha_{i}$-expansive and $B_{i}$ is $\beta_{i}$-Lipschitz continuous, $i=$ 1,2 .

(iii) $H_{i}\left(A_{i}, B_{i}\right)$ is $r_{i}$-Lipschitz continuous with respect to $A_{i}$ and $s_{i}$-Lipschitz continuous with respect to $B_{i}, i=1,2$.

(iv) $T$ is D-Lipschitz continuous with constant $\lambda_{T}$ and $Q$ is D-Lipschitz continuous with constant $\lambda_{Q}$.

(v) $F$ is $t_{1}$-Lipschitz continuous with respect to $T$ in the first argument and $t_{2}$-Lipschitz continuous with respect to $Q$ in the second argument.

(vi) $G$ is $l_{1}$-Lipschitz continuous with respect to $T$ in the first argument and $l_{2}$-Lipschitz continuous with respect to $Q$ in the second argument.

(vii) $\eta_{i}$ is $\rho_{i}$-Lipschitz continuous, $i=1,2$.

(viii) $F$ is $\eta_{1}$-relaxed Lipschitz continuous with respect to $T$ in the first argument and $\eta_{1}$-relaxed Lipschitz continuous with respect to $Q$ in the second argument with constants $\tau_{1}$ and $\tau_{2}$, respectively.

(ix) G is $\eta_{2}$-relaxed Lipschitz continuous with respect to $T$ in the first argument and $\eta_{2}$-relaxed Lipschitz continuous with respect to $Q$ in the second argument with constants $\epsilon_{1}$ and $\epsilon_{2}$, respectively. Furthermore, assume that there exist constants $\sigma_{1}, \sigma_{2}>0$ such that

$$
\begin{aligned}
& \left\|R_{\lambda_{1}, M\left(\cdot, x_{1}\right)}^{H_{1}(\cdot,)-\eta_{1}}(x)-R_{\lambda_{1}, M\left(\cdot, x_{2}\right)}^{H_{1}(\cdot, \cdot)-\eta_{1}}(x)\right\|_{1} \\
& \quad \leq \sigma_{1}\left\|x_{1}-x_{2}\right\|_{1}, \quad \forall x, x_{1}, x_{2} \in X_{1}, \\
& \left\|R_{\lambda_{2}, N\left(\cdot, y_{1}\right)}^{H_{2}(\cdot,)-\eta_{2}}(y)-R_{\lambda_{2}, N\left(\cdot, y_{2}\right)}^{H_{2}(\cdot,)-\eta_{2}}(y)\right\|_{2} \\
& \quad \leq \sigma_{2}\left\|y_{1}-y_{2}\right\|_{2}, \quad \forall y, y_{1}, y_{2} \in X_{2},
\end{aligned}
$$

and $\lambda_{1}, \lambda_{2}>0$ are constants satisfying the following conditions:

$$
\begin{aligned}
p_{0}= & \sqrt{r_{1}^{2}+2 \lambda_{1} t_{1} \lambda_{T}\left[r_{1}+\lambda_{1} t_{1} \lambda_{T}+\rho_{1}\right]-2 \lambda_{1} \tau_{1}}, \\
q_{0}= & \sqrt{r_{2}^{2}+2 \lambda_{2} l_{2} \lambda_{Q}\left[r_{2}+\lambda_{2} l_{2} \lambda_{Q}+\rho_{2}\right]-2 \lambda_{2} \epsilon_{2}}, \\
& r_{1}^{2}+2 \lambda_{1} t_{1} \lambda_{T}\left[r_{1}+\lambda_{1} t_{1} \lambda_{T}+\rho_{1}\right]>2 \lambda_{1} \tau_{1}, \\
& r_{2}^{2}+2 \lambda_{2} l_{2} \lambda_{Q}\left[r_{2}+\lambda_{2} l_{2} \lambda_{Q}+\rho_{2}\right]>2 \lambda_{2} \epsilon_{2},
\end{aligned}
$$

$$
\begin{gathered}
\mu_{i}>\gamma_{i}, \quad \alpha_{i}>\beta_{i}, \quad i=1,2, \\
\theta_{1}=\frac{\rho_{1}}{\mu_{1} \alpha_{1}^{2}-\gamma_{1} \beta_{1}^{2}}, \quad \theta_{2}=\frac{\rho_{2}}{\mu_{2} \alpha_{2}^{2}-\gamma_{2} \beta_{2}^{2}}, \\
\sigma_{1}+\theta_{1} p_{0}+\theta_{1} s_{1}+\lambda_{2} \theta_{2} l_{1} \lambda_{T}<1, \\
\sigma_{2}+\theta_{2} q_{0}+\theta_{2} s_{2}+\lambda_{1} \theta_{1} t_{2} \lambda_{Q}<1 .
\end{gathered}
$$

Then, the iterative sequences $\left\{x_{n}\right\},\left\{y_{n}\right\},\left\{w_{n}\right\}$, and $\left\{v_{n}\right\}$ generated by Algorithm 17 converge strongly to $x, y, w$, and $v$, respectively, and $(x, y, w, v)$ is a solution of problem (30).

Proof. In view of Theorem 13, the resolvent operator $R_{\lambda_{1}, M}^{H_{1}(\cdot, \cdot)-\eta_{1}}$ is $\theta_{1}$-Lipschitz continuous. This, together with Algorithm 17 and (36), implies that

$$
\begin{aligned}
& \left\|x_{n+1}-x_{n}\right\|_{1} \\
& =\| R_{\lambda_{1}, M\left(\cdot, x_{n}\right)}^{H_{1}(\cdot,)-\eta_{1}}\left[H_{1}\left(A_{1} x_{n}, B_{1} x_{n}\right)-\lambda_{1} F\left(w_{n}, v_{n}\right)\right] \\
& -R_{\lambda_{1}, M\left(\cdot, x_{n-1}\right)}^{H_{1}(\cdot,)-\eta_{1}} \\
& \times\left[H_{1}\left(A_{1} x_{n-1}, B_{1} x_{n-1}\right)-\lambda_{1} F\left(w_{n-1}, v_{n-1}\right)\right] \|_{1} \\
& \leq \| R_{\lambda_{1}, M\left(\cdot, x_{n}\right)}^{H_{1}(\cdot \cdot)-\eta_{1}}\left[H_{1}\left(A_{1} x_{n}, B_{1} x_{n}\right)-\lambda_{1} F\left(w_{n}, v_{n}\right)\right] \\
& -R_{\lambda_{1}, M\left(\cdot, x_{n-1}\right)}^{H_{1}(\cdot,)-\eta_{1}}\left[H_{1}\left(A_{1} x_{n}, B_{1} x_{n}\right)-\lambda_{1} F\left(w_{n}, v_{n}\right)\right] \|_{1} \\
& +\| R_{\lambda_{1}, M\left(\cdot, x_{n-1}\right)}^{H_{1}(\cdot \cdot)-\eta_{1}}\left[H_{1}\left(A_{1} x_{n}, B_{1} x_{n}\right)-\lambda_{1} F\left(w_{n}, v_{n}\right)\right] \\
& -R_{\lambda_{1}, M\left(\cdot, x_{n-1}\right)}^{H_{1}(\cdot,)-\eta_{1}} \\
& \times\left[H_{1}\left(A_{1} x_{n-1}, B_{1} x_{n-1}\right)-\lambda_{1} F\left(w_{n-1}, v_{n-1}\right)\right] \|_{1} \\
& \leq \sigma_{1}\left\|x_{n}-x_{n-1}\right\|_{1} \\
& +\theta_{1} \| H_{1}\left(A_{1} x_{n}, B_{1} x_{n}\right)-H_{1}\left(A_{1} x_{n-1}, B_{1} x_{n-1}\right) \\
& -\lambda_{1}\left(F\left(w_{n}, v_{n}\right)-F\left(w_{n-1}, v_{n-1}\right)\right) \|_{1} \\
& \leq \sigma_{1}\left\|x_{n}-x_{n-1}\right\|_{1} \\
& +\theta_{1} \| H_{1}\left(A_{1} x_{n}, B_{1} x_{n}\right)-H_{1}\left(A_{1} x_{n-1}, B_{1} x_{n-1}\right) \\
& -\lambda_{1}\left(F\left(w_{n}, v_{n}\right)-F\left(w_{n-1}, v_{n}\right)\right) \|_{1} \\
& +\lambda_{1} \theta_{1}\left\|F\left(w_{n-1}, v_{n}\right)-F\left(w_{n-1}, v_{n-1}\right)\right\|_{1} \\
& \leq \sigma_{1}\left\|x_{n}-x_{n-1}\right\|_{1} \\
& +\theta_{1} \| H_{1}\left(A_{1} x_{n}, B_{1} x_{n}\right)-H_{1}\left(A_{1} x_{n-1}, B_{1} x_{n}\right) \\
& -\lambda_{1}\left(F\left(w_{n}, v_{n}\right)-F\left(w_{n-1}, v_{n}\right)\right) \|_{1}
\end{aligned}
$$




$$
\begin{aligned}
& +\theta_{1}\left\|H_{1}\left(A_{1} x_{n-1}, B_{1} x_{n}\right)-H_{1}\left(A_{1} x_{n-1}, B_{1} x_{n-1}\right)\right\|_{1} \\
& +\lambda_{1} \theta_{1}\left\|F\left(w_{n-1}, v_{n}\right)-F\left(w_{n-1}, v_{n-1}\right)\right\|_{1} .
\end{aligned}
$$

Since $F$ is $t_{1}$-Lipschitz continuous with respect to $T$ in the first argument and $t_{2}$-Lipschitz continuous in the second argument, $T$ is $\lambda_{T}$-Lipschitz continuous, and $Q$ is $\lambda_{Q^{-}}$ Lipschitz continuous, by Algorithm 17, we get

$$
\begin{aligned}
\| F & \left(w_{n}, v_{n}\right)-F\left(w_{n-1}, v_{n}\right) \|_{1} \\
& \leq t_{1}\left\|w_{n}-w_{n-1}\right\|_{1} \\
& \leq t_{1}\left(1+\frac{1}{n}\right) D\left(T\left(x_{n}\right), T\left(x_{n-1}\right)\right) \\
& \leq t_{1} \lambda_{T}\left(1+\frac{1}{n}\right)\left\|x_{n}-x_{n-1}\right\|_{1}, \\
\| F & \left(w_{n-1}, v_{n}\right)-F\left(w_{n-1}, v_{n-1}\right) \|_{1} \\
& \leq t_{2}\left\|v_{n}-v_{n-1}\right\|_{2} \\
& \leq t_{2}\left(1+\frac{1}{n}\right) D\left(Q\left(y_{n}\right), Q\left(y_{n-1}\right)\right) \\
& \leq t_{2} \lambda_{Q}\left(1+\frac{1}{n}\right)\left\|y_{n}-y_{n-1}\right\|_{2} .
\end{aligned}
$$

As $H_{1}(\cdot, \cdot)$ is $r_{1}$-Lipschitz continuous with respect to $A_{1}$, we obtain

$$
\left\|H_{1}\left(A_{1} x_{n}, B_{1} x_{n}\right)-H_{1}\left(A_{1} x_{n-1}, B_{1} x_{n}\right)\right\|_{1} \leq r_{1}\left\|x_{n}-x_{n-1}\right\|_{1} .
$$

Since $\eta_{1}$ is $\rho_{1}$-Lipschitz continuous, we conclude that

$$
\left\|\eta_{1}\left(x_{n}, x_{n-1}\right)\right\|_{1} \leq \rho_{1}\left\|x_{n}-x_{n-1}\right\|_{1} .
$$

Since $H_{1}(\cdot, \cdot)$ is $\eta_{1}$-relaxed Lipschitz continuous with respect to $T$ and $\eta_{2}$-relaxed Lipschitz continuous with respect to $Q$ in the first and second arguments with constants $\tau_{1}$ and $\tau_{2}$, respectively, we have

$$
\begin{aligned}
& \left\langle F\left(w_{n}, v_{n}\right)-F\left(w_{n-1}, v_{n}\right), j\left(\eta_{1}\left(x_{n}, x_{n-1}\right)\right)\right\rangle \\
& \quad \leq-\tau_{1}\left\|x_{n}-x_{n-1}\right\|_{1}^{2} .
\end{aligned}
$$

Employing Lemma 3 and taking into account (39)-(44), we obtain

$$
\begin{aligned}
\| H_{1} & \left(A_{1} x_{n}, B_{1} x_{n}\right)-H_{1}\left(A_{1} x_{n-1}, B_{1} x_{n}\right) \\
- & \lambda_{1}\left(F\left(w_{n}, v_{n}\right)-F\left(w_{n-1}, v_{n}\right)\right) \|_{1}^{2} \\
\leq & \left\|H_{1}\left(A_{1} x_{n}, B_{1} x_{n}\right)-H_{1}\left(A_{1} x_{n-1}, B_{1} x_{n}\right)\right\|_{1}^{2} \\
& -2 \lambda_{1}\left\langle F\left(w_{n}, v_{n}\right)-F\left(w_{n-1}, v_{n}\right),\right.
\end{aligned}
$$

$$
\begin{aligned}
& j\left[H_{1}\left(A_{1} x_{n}, B_{1} x_{n}\right)-H_{1}\left(A_{1} x_{n-1}, B_{1} x_{n}\right)\right. \\
& \left.\left.-\lambda_{1}\left(F\left(w_{n}, v_{n}\right)-F\left(w_{n-1}, v_{n}\right)\right)\right]\right\rangle \\
& =\left\|H_{1}\left(A_{1} x_{n}, B_{1} x_{n}\right)-H_{1}\left(A_{1} x_{n-1}, B_{1} x_{n}\right)\right\|_{1}^{2} \\
& -2 \lambda_{1}\left\langle F\left(w_{n}, v_{n}\right)-F\left(w_{n-1}, v_{n}\right)\right. \text {, } \\
& j\left[H_{1}\left(A_{1} x_{n}, B_{1} x_{n}\right)-H_{1}\left(A_{1} x_{n-1}, B_{1} x_{n}\right)\right. \\
& \left.-\lambda_{1}\left(F\left(w_{n}, v_{n}\right)-F\left(w_{n-1}, v_{n}\right)\right)\right] \\
& \left.+j\left(\eta_{1}\left(x_{n}, x_{n-1}\right)\right)\right\rangle \\
& +2 \lambda_{1}\left\langle F\left(w_{n}, v_{n}\right)-F\left(w_{n-1}, v_{n}\right), j\left(\eta_{1}\left(x_{n}, x_{n-1}\right)\right)\right\rangle \\
& \leq\left\|H_{1}\left(A_{1} x_{n}, B_{1} x_{n}\right)-H_{1}\left(A_{1} x_{n-1}, B_{1} x_{n}\right)\right\|_{1}^{2} \\
& +2 \lambda_{1}\left\|F\left(w_{n}, v_{n}\right)-F\left(w_{n-1}, v_{n}\right)\right\|_{1} \\
& \times\left[\left\|H_{1}\left(A_{1} x_{n}, B_{1} x_{n}\right)-H_{1}\left(A_{1} x_{n-1}, B_{1} x_{n}\right)\right\|_{1}\right. \\
& +\lambda_{1}\left\|F\left(w_{n}, v_{n}\right)-F\left(w_{n-1}, v_{n}\right)\right\|_{1} \\
& \left.+\left\|\eta_{1}\left(x_{n}, x_{n-1}\right)\right\|_{1}\right] \\
& +2 \lambda_{1}\left\langle F\left(w_{n}, v_{n}\right)-F\left(w_{n-1}, v_{n}\right), j\left(\eta_{1}\left(x_{n}, x_{n-1}\right)\right)\right\rangle \\
& \leq r_{1}^{2}\left\|x_{n}-x_{n-1}\right\|_{1}^{2}+2 \lambda_{1} t_{1} \lambda_{T}\left(1+\frac{1}{n}\right)\left\|x_{n}-x_{n-1}\right\|_{1} \\
& \times\left[r_{1}\left\|x_{n}-x_{n-1}\right\|_{1}+\lambda_{1} t_{1} \lambda_{T}\left(1+\frac{1}{n}\right)\right. \\
& \left.\times\left\|x_{n}-x_{n-1}\right\|_{1}+\rho_{1}\left\|x_{n}-x_{n-1}\right\|_{1}\right] \\
& -2 \lambda_{1} \tau_{1}\left\|x_{n}-x_{n-1}\right\|_{1}^{2} \\
& =\left[r_{1}^{2}+2 \lambda_{1} t_{1} \lambda_{T}\left(1+\frac{1}{n}\right)\right. \\
& \left.\times\left[r_{1}+\lambda_{1} t_{1} \lambda_{T}\left(1+\frac{1}{n}\right)+\rho_{1}\right]-2 \lambda_{1} \tau_{1}\right]\left\|x_{n}-x_{n-1}\right\|_{1}^{2} .
\end{aligned}
$$

This implies that

$$
\begin{aligned}
& \| H_{1}\left(A_{1} x_{n}, B_{1} x_{n}\right)-H_{1}\left(A_{1} x_{n-1}, B_{1} x_{n}\right) \\
& -\lambda_{1}\left(F\left(w_{n}, v_{n}\right)-F\left(w_{n-1}, v_{n}\right)\right) \|_{1} \\
& \leq \sqrt{r_{1}^{2}+2 \lambda_{1} t_{1} \lambda_{T}\left(1+\frac{1}{n}\right)\left[r_{1}+\lambda_{1} t_{1} \lambda_{T}\left(1+\frac{1}{n}\right)+\rho_{1}\right]-2 \lambda_{1} \tau_{1}} \\
& \quad \times\left\|x_{n}-x_{n-1}\right\|_{1} \\
& =p_{n}\left\|x_{n}-x_{n-1}\right\|_{1},
\end{aligned}
$$

where

$$
\begin{aligned}
& p_{n} \\
& =\sqrt{r_{1}^{2}+2 \lambda_{1} t_{1} \lambda_{T}\left(1+\frac{1}{n}\right)\left[\left[r_{1}+\lambda_{1} t_{1} \lambda_{T}\left(1+\frac{1}{n}\right)+\rho_{1}\right]-2 \lambda_{1} \tau_{1}\right]} .
\end{aligned}
$$


Using $s_{1}$-Lipschitz continuity of $H_{1}(\cdot, \cdot)$ with respect to $B_{1}$, we deduce that

$$
\begin{aligned}
& \left\|H_{1}\left(A_{1} x_{n-1}, B_{1} x_{n}\right)-H_{1}\left(A_{1} x_{n-1}, B_{1} x_{n-1}\right)\right\|_{1} \\
& \quad \leq s_{1}\left\|x_{n}-x_{n-1}\right\|_{1} .
\end{aligned}
$$

In view of (41), (46), (48), (39) becomes

$$
\begin{aligned}
& \left\|x_{n+1}-x_{n}\right\|_{1} \\
& \begin{aligned}
\leq \sigma_{1}\left\|x_{n}-x_{n-1}\right\|_{1} & +\theta_{1} p_{n}\left\|x_{n}-x_{n-1}\right\|_{1} \\
& +\theta_{1} s_{1}\left\|x_{n}-x_{n-1}\right\|_{1} \\
& +\lambda_{1} \theta_{1} t_{2} \lambda_{\mathrm{Q}}\left(1+\frac{1}{n}\right)\left\|y_{n}-y_{n-1}\right\|_{2} \\
= & \left(\sigma_{1}+\theta_{1} p_{n}+\theta_{1} s_{1}\right)\left\|x_{n}-x_{n-1}\right\|_{1} \\
& +\lambda_{1} \theta_{1} t_{2} \lambda_{\mathrm{Q}}\left(1+\frac{1}{n}\right)\left\|y_{n}-y_{n-1}\right\|_{2} .
\end{aligned}
\end{aligned}
$$

Similarly, we have

$$
\begin{aligned}
& \left\|y_{n+1}-y_{n}\right\|_{2} \\
& =\| R_{\lambda_{2}, N\left(\cdot, y_{n}\right)}^{H_{2}(\cdot,)-\eta_{2}}\left[H_{2}\left(A_{2} y_{n}, B_{2} y_{n}\right)-\lambda_{2} G\left(w_{n}, v_{n}\right)\right] \\
& -R_{\lambda_{2}, N\left(\cdot, y_{n-1}\right)}^{H_{2}(\cdot,) \eta_{2}}\left[H_{2}\left(A_{2} y_{n-1}, B_{2} y_{n-1}\right)\right. \\
& \left.-\lambda_{2} G\left(w_{n-1}, v_{n-1}\right)\right] \|_{2} \\
& \leq \| R_{\lambda_{2}, N\left(\cdot, y_{n}\right)}^{H_{2}(\cdot,)-\eta_{2}}\left[H_{2}\left(A_{2} y_{n}, B_{2} y_{n}\right)-\lambda_{2} G\left(w_{n}, v_{n}\right)\right] \\
& -R_{\lambda_{2}, N\left(\cdot, y_{n-1}\right)}^{H_{2}(\cdot,), \eta_{2}}\left[H_{2}\left(A_{2} y_{n}, B_{2} y_{n}\right)-\lambda_{2} G\left(w_{n}, v_{n}\right)\right] \|_{2} \\
& +\| R_{\lambda_{2}, N\left(\cdot, y_{n-1}\right)}^{H_{2}(\cdot,) \eta_{2}}\left[H_{2}\left(A_{2} y_{n}, B_{2} y_{n}\right)-\lambda_{2} G\left(w_{n}, v_{n}\right)\right] \\
& -R_{\lambda_{2}, N\left(\cdot, y_{n-1}\right)}^{H_{2}(\cdot,)-\eta_{2}}\left[H_{2}\left(A_{2} y_{n-1}, B_{2} y_{n-1}\right)\right. \\
& \left.-\lambda_{2} G\left(w_{n-1}, v_{n-1}\right)\right] \|_{2} \\
& \leq \sigma_{2}\left\|y_{n}-y_{n-1}\right\|_{2} \\
& +\theta_{2} \| H_{2}\left(A_{2} y_{n}, B_{2} y_{n}\right)-H_{2}\left(A_{2} y_{n-1}, B_{2} y_{n-1}\right) \\
& -\lambda_{2}\left(G\left(w_{n}, v_{n}\right)-G\left(w_{n-1}, v_{n-1}\right)\right) \|_{2} \\
& \leq \sigma_{2}\left\|y_{n}-y_{n-1}\right\|_{2} \\
& +\theta_{2} \| H_{2}\left(A_{2} y_{n}, B_{2} y_{n}\right)-H_{2}\left(A_{2} y_{n-1}, B_{2} y_{n-1}\right) \\
& \text { - } \lambda_{2}\left(G\left(w_{n}, v_{n}\right)-G\left(w_{n}, v_{n-1}\right)\right) \|_{2} \\
& +\lambda_{2} \theta_{2}\left\|G\left(w_{n}, v_{n-1}\right)-G\left(w_{n-1}, v_{n-1}\right)\right\|_{2}
\end{aligned}
$$

$$
\begin{aligned}
\leq & \sigma_{2}\left\|y_{n}-y_{n-1}\right\|_{2} \\
& +\theta_{2} \| H_{2}\left(A_{2} y_{n}, B_{2} y_{n}\right)-H_{2}\left(A_{2} y_{n-1}, B_{2} y_{n}\right) \\
& \quad-\lambda_{2}\left(G\left(w_{n}, v_{n}\right)-G\left(w_{n}, v_{n-1}\right)\right) \|_{2} \\
& +\theta_{2}\left\|H_{2}\left(A_{2} y_{n-1}, B_{2} y_{n}\right)-H_{2}\left(A_{2} y_{n-1}, B_{2} y_{n-1}\right)\right\|_{2} \\
& +\lambda_{2} \theta_{2}\left\|G\left(w_{n}, v_{n-1}\right)-G\left(w_{n-1}, v_{n-1}\right)\right\|_{2} \\
\leq & \sigma_{2}\left\|y_{n}-y_{n-1}\right\|_{2}+\theta_{2} q_{n}\left\|y_{n}-y_{n-1}\right\|_{2}+\theta_{2} s_{2}\left\|y_{n}-y_{n-1}\right\|_{2} \\
& +\lambda_{2} \theta_{2} t_{1} \lambda_{T}\left(1+\frac{1}{n}\right)\left\|x_{n}-x_{n-1}\right\|_{1} \\
= & \left(\sigma_{2}+\theta_{2} q_{n}+\theta_{2} s_{2}\right)\left\|y_{n}-y_{n-1}\right\|_{2} \\
& +\lambda_{2} \theta_{2} l_{1} \lambda_{T}\left(1+\frac{1}{n}\right)\left\|x_{n}-x_{n-1}\right\|_{1},
\end{aligned}
$$

where

$$
=\sqrt{r_{2}^{2}+2 \lambda_{2} l_{2} \lambda_{Q}\left(1+\frac{1}{n}\right)\left[r_{2}+\lambda_{2} l_{2} \lambda_{Q}\left(1+\frac{1}{n}\right)+\rho_{2}\right]-2 \lambda_{2} \epsilon_{2}} .
$$

In view of (49) and (50), we obtain

$$
\begin{aligned}
& \left\|x_{n+1}-x_{n}\right\|_{1}+\left\|y_{n+1}-y_{n}\right\|_{2} \\
& \quad \leq c_{n}\left\|x_{n}-x_{n-1}\right\|_{1}+d_{n}\left\|y_{n}-y_{n-1}\right\|_{2} \\
& \quad \leq k_{n}\left(\left\|x_{n}-x_{n-1}\right\|_{1}+\left\|y_{n}-y_{n-1}\right\|_{2}\right),
\end{aligned}
$$

where $c_{n}=\sigma_{1}+\theta_{1} p_{n}+\theta_{1} s_{1}+\lambda_{2} \theta_{2} l_{1} \lambda_{T}(1+1 / n), d_{n}=\sigma_{2}+$ $\theta_{2} q_{n}+\theta_{2} s_{2}+\lambda_{1} \theta_{1} t_{2} \lambda_{Q}(1+1 / n)$, and $k_{n}=\max \left\{c_{n}, d_{n}\right\}$.

Letting $n \rightarrow \infty$, we obtain $k_{n} \rightarrow k$, where

$$
\begin{array}{r}
k=\max \left\{\sigma_{1}+\theta_{1} p_{0}+\theta_{1} s_{1}+\lambda_{2} \theta_{2} l_{1} \lambda_{T},\right. \\
\left.\sigma_{2}+\theta_{2} q_{0}+\theta_{2} s_{2}+\lambda_{1} \theta_{1} t_{2} \lambda_{\mathrm{Q}}\right\} .
\end{array}
$$

Next, we define the norm $\|\cdot\|$ on $X_{1} \times X_{2}$ by

$$
\|(x, y)\|=\|x\|_{1}+\|y\|_{2}, \quad(x, y) \in X_{1} \times X_{2} .
$$

One can easily check that $\left(X_{1} \times X_{2},\|\cdot\|\right)$ is a Banach space.

Define $a_{n+1}=\left(x_{n+1}, y_{n+1}\right)$. Then, we have

$$
\left\|a_{n+1}-a_{n}\right\|=\left\|x_{n+1}-x_{n}\right\|_{1}+\left\|y_{n+1}-y_{n}\right\|_{2} .
$$

In view of (38), we conclude that $0<k<1$. This implies that there exist $n_{0} \in \mathbb{N}$ and $k_{0} \in(0,1)$ such that $k_{n} \leq k_{0}$ for all $n \geq n_{0}$. It follows from (52) and (54) that

$$
\left\|a_{n+1}-a_{n}\right\| \leq k_{0}\left\|a_{n}-a_{n-1}\right\|, \quad \forall n \geq n_{0} .
$$

In view of (56), we obtain

$$
\left\|a_{n+1}-a_{n}\right\| \leq k_{0}^{n-n_{0}}\left\|a_{n_{0}+1}-a_{n_{0}}\right\|, \quad \forall n \geq n_{0} .
$$


This implies that for any $m \geq n \geq n_{0}$,

$$
\begin{aligned}
& \left\|x_{m}-x_{n}\right\|_{1} \leq\left\|a_{m}-a_{n}\right\| \\
& \quad \leq \sum_{i=n}^{m-1}\left\|a_{i+1}-a_{i}\right\| \leq \sum_{i=n}^{m-1} k_{0}^{i-n_{0}}\left\|a_{n_{0}+1}-a_{n_{0}}\right\| .
\end{aligned}
$$

Since $0<k_{0}<1$, it follows from (58) that $\left\|x_{m}-x_{n}\right\|_{1} \rightarrow 0$ and $n \rightarrow \infty$. This proves that $\left\{x_{n}\right\}$ is a Cauchy sequence in $X_{1}$. Similarly, we conclude that $\left\{y_{n}\right\}$ is a Cauchy sequence in $X_{2}$. Thus, there exist $x \in X_{1}$ and $y \in X_{2}$ such that $x_{n} \rightarrow x$ and $y_{n} \rightarrow y$ as $n \rightarrow \infty$.

Next, we prove that $w_{n} \rightarrow w \in T(x)$ and $v_{n} \rightarrow v \in Q(y)$. In view of Lipschitz continuity of $T$ and $Q$ and Algorithm 17, we obtain

$$
\begin{aligned}
& \left\|w_{n}-w_{n-1}\right\|_{1} \leq\left(1+\frac{1}{n}\right) \lambda_{T}\left\|x_{n}-x_{n-1}\right\|_{1}, \\
& \left\|v_{n}-v_{n-1}\right\|_{2} \leq\left(1+\frac{1}{n}\right) \lambda_{\mathrm{Q}}\left\|y_{n}-y_{n-1}\right\|_{2} .
\end{aligned}
$$

From (59), we deduce that $\left\{w_{n}\right\},\left\{v_{n}\right\}$ are Cauchy sequences in $X_{1}$ and $X_{2}$, respectively. Thus, there exist $w \in T(x)$ and $v \in Q(y)$ such that $w_{n} \rightarrow w$ and $v_{n} \rightarrow v$ as $n \rightarrow \infty$. Since $T$ is $D$-Lipschitz continuous with constant $\lambda_{T}$, it is obvious that

$$
\begin{aligned}
d(w, T(x)) \leq & \left\|w-w_{n}\right\|_{1}+d\left(w_{n}, T(x)\right) \\
\leq & \left\|w-w_{n-1}\right\|_{1}+D\left(T\left(x_{n}\right), T(x)\right) \\
\leq & \left\|w-w_{n}\right\|_{1} \\
& \quad+\lambda_{T}\left\|x_{n}-x\right\|_{1} \longrightarrow 0(n \longrightarrow \infty) .
\end{aligned}
$$

By the closedness of $T(x)$, we conclude that $w \in T(x)$. Similarly, we have $v \in Q(y)$.

Assume now that

$$
\begin{aligned}
& x_{0}=R_{\lambda_{1}, M(\cdot, x)}^{H_{1}(\cdot \cdot)-\eta}\left[H_{1}\left(A_{1} x, B_{1} x\right)-\lambda_{1} F(w, v)\right], \\
& y_{0}=R_{\lambda_{2}, N(\cdot, y)}^{H_{2}(\cdot, \cdot)-\eta}\left[H_{2}\left(A_{2} y, B_{2} y\right)-\lambda_{2} G(w, v)\right] .
\end{aligned}
$$

Then, we have

$$
\begin{aligned}
& \left\|x_{n+1}-x_{0}\right\|_{1} \\
& \leq \| R_{\lambda_{1}, M\left(\cdot, x_{n}\right)}^{H_{1}(\cdot,)-\eta_{1}}\left[H_{1}\left(A_{1} x_{n}, B_{1} x_{n}\right)-\lambda_{1} F\left(w_{n}, v_{n}\right)\right] \\
& \quad-R_{\lambda_{1}, M(\cdot, x)}^{H_{1}(\cdot,)-\eta_{1}}\left[H_{1}\left(A_{1} x, B_{1} x\right)-\lambda_{1} F(w, v)\right] \|_{1} \\
& \leq \| R_{\lambda_{1}, M\left(\cdot, x_{n}\right)}^{H_{1}(\cdot, \cdot)-\eta_{1}}\left[H_{1}\left(A_{1} x_{n}, B_{1} x_{n}\right)-\lambda_{1} F\left(w_{n}, v_{n}\right)\right] \\
& \quad-R_{\lambda_{1}, M(\cdot, x)}^{H_{1}(\cdot,)-\eta_{1}}\left[H_{1}\left(A_{1} x_{n}, B_{1} x_{n}\right)-\lambda_{1} F\left(w_{n}, v_{n}\right)\right] \|_{1}
\end{aligned}
$$

$$
\begin{aligned}
& +\| R_{\lambda_{1}, M(\cdot, x)}^{H_{1}(\cdot \cdot)-\eta_{1}}\left[H_{1}\left(A_{1} x_{n}, B_{1} x_{n}\right)-\lambda_{1} F\left(w_{n}, v_{n}\right)\right] \\
& -R_{\lambda_{1}, M(\cdot, x)}^{H_{1}(\cdot, \cdot)-\eta_{1}}\left[H_{1}\left(A_{1} x, B_{1} x\right)-\lambda_{1} F(w, v)\right] \|_{1} \\
& \leq \sigma_{1}\left\|x_{n}-x\right\|_{1} \\
& +\theta_{1} \| H_{1}\left(A_{1} x_{n}, B_{1} x_{n}\right)-\lambda_{1} F\left(w_{n}, v_{n}\right) \\
& -\left[H_{1}\left(A_{1} x, B_{1} x\right)-\lambda_{1} F(w, v)\right] \|_{1} \\
& \leq \sigma_{1}\left\|x_{n}-x\right\|_{1} \\
& +\theta_{1}\left[\left\|H_{1}\left(A_{1} x_{n}, B_{1} x_{n}\right)-H_{1}\left(A_{1} x, B_{1} x\right)\right\|_{1}\right. \\
& \left.+\lambda_{1}\left\|F\left(w_{n}, v_{n}\right)-F(w, v)\right\|_{1}\right] \\
& \leq \sigma_{1}\left\|x_{n}-x\right\|_{1} \\
& +\theta_{1}\left[\left\|H_{1}\left(A_{1} x_{n}, B_{1} x_{n}\right)-H_{1}\left(A_{1} x, B_{1} x_{n}\right)\right\|_{1}\right. \\
& \left.+\left\|H_{1}\left(A_{1} x, B_{1} x_{n}\right)-H_{1}\left(A_{1} x, B_{1} x\right)\right\|_{1}\right] \\
& +\lambda_{1} \theta_{1}\left[\left\|F\left(w_{n}, v_{n}\right)-F\left(w, v_{n}\right)\right\|_{1}\right. \\
& \left.+\left\|F\left(w, v_{n}\right)-F(w, v)\right\|_{1}\right] \\
& \leq \sigma_{1}\left\|x_{n}-x\right\|_{1}+\theta_{1}\left[r_{1}+s_{1}\right]\left\|x_{n}-x\right\|_{1} \\
& +\lambda_{1} \theta_{1}\left[t_{1}\left\|w_{n}-w\right\|_{1}+t_{2}\left\|v_{n}-v\right\|_{2}\right] \\
& =\left(\sigma_{1}+\theta_{1} r_{1}+\theta_{1} s_{1}\right)\left\|x_{n}-x\right\|_{1} \\
& +\lambda_{1} \theta_{1}\left[t_{1}\left\|w_{n}-w\right\|_{1}+t_{2}\left\|v_{n}-v\right\|_{2}\right] \text {. }
\end{aligned}
$$

Since $x_{n} \rightarrow x, w_{n} \rightarrow w$, and $v_{n} \rightarrow v$ as $n \rightarrow \infty$, it follows from (62) that

$$
\lim _{n \rightarrow \infty}\left\|x_{n}-x_{0}\right\|_{1}=0 \text {, }
$$

and hence $x_{0}=x$.

A similar argument shows that $y_{0}=y$. Therefore,

$$
\begin{aligned}
& x=x_{0}=R_{\lambda_{1}, M(\cdot, x)}^{H_{1}(\cdot, \cdot)-\eta}\left[H_{1}\left(A_{1} x, B_{1} x\right)-\lambda_{1} F(w, v)\right], \\
& y=y_{0}=R_{\lambda_{2}, N(\cdot, y)}^{H_{2}(\cdot, \cdot)-\eta}\left[H_{2}\left(A_{2} y, B_{2} y\right)-\lambda_{2} G(w, v)\right] .
\end{aligned}
$$

In view of Lemma 16, we conclude that $(x, y, w, v)$ is a solution of problem (30), which completes the proof.

At the end of this paper, we include the following simple example in support of Theorem 18.

Example 19. Let $X=\mathbb{R}^{2}$ with the usual inner product. We define two mappings $A, B: \mathbb{R}^{2} \rightarrow \mathbb{R}^{2}$ by

$$
\begin{array}{r}
A(x):=\left(\frac{1}{4} x_{1}-x_{2}, x_{1}+\frac{1}{4} x_{2}\right) \\
B(x):=\left(-\frac{1}{4} x_{1}+\frac{1}{4} x_{2},-\frac{1}{4} x_{1}-\frac{1}{4} x_{2}\right), \\
\forall x=\left(x_{1}, x_{2}\right) \in \mathbb{R}^{2} .
\end{array}
$$


Let a mapping $H: \mathbb{R}^{2} \times \mathbb{R}^{2} \rightarrow \mathbb{R}^{2}$ be defined by

$$
H(A x, B y):=A x+B y, \quad \forall x, y \in \mathbb{R}^{2} .
$$

By similar arguments, as in Example 4.1 of [27], we can prove the following.

(1) $H(A, B)$ is $4 / 17$-cocoercive with respect to $A$ and 1 relaxed cocoercive with respect to $B$.

(2) $A$ is $\sqrt{17} / n$-expansive, for $n=4,5$.

(3) $B$ is $1 / \sqrt{n}$-expansive, for $n=1,2$.

(4) $H(A, B)$ is $\sqrt{17} / n$-Lipschitz continuous with constant $\sqrt{17} / n$ with respect to $A$ and $B$, for $n=1,2, \ldots, 15,16$.

(5) Let $f, g: \mathbb{R}^{2} \rightarrow \mathbb{R}^{2}$ be defined by

$$
\begin{array}{r}
f(x):=\left(8 x_{1}-\frac{8}{5} x_{2}, \frac{8}{5} x_{1}+8 x_{2}\right), \\
g(x):=\left(-\frac{17}{8} x_{1}+\frac{5}{8} x_{2}, \frac{5}{8} x_{1}+\frac{17}{8} x_{2}\right), \\
\forall x=\left(x_{1}, x_{2}\right) \in \mathbb{R}^{2} .
\end{array}
$$

(6) Now, we define a mapping $M: \mathbb{R}^{2} \times \mathbb{R}^{2} \rightarrow \mathbb{R}^{2}$ by

$$
M(f x, g y):=f x-g y, \quad \forall x, y \in \mathbb{R}^{2}
$$

Let $R, S, T: \mathbb{R}^{2} \rightarrow \mathbb{R}^{2}$ be the identity mappings. It is obvious that these mappings are $D$-Lipschitz continuous.

(7) Assume that $F, G: \mathbb{R}^{2} \rightarrow \mathbb{R}^{2}$ are defined by

$$
\begin{array}{r}
F(x):=\left(-\frac{1}{4} x_{1}-\frac{1}{8} x_{2}, \frac{1}{8} x_{1}-\frac{1}{4} x_{2}\right), \\
G(x):=\left(-\frac{1}{8} x_{1}+\frac{1}{5} x_{2},-\frac{1}{5} x_{1}-\frac{1}{8} x_{2}\right), \\
\forall x=\left(x_{1}, x_{2}\right) \in \mathbb{R}^{2} .
\end{array}
$$

It could easily be seen that all the aspects of the hypotheses of Theorem 18 are satisfied, so we have the desired conclusion.

\section{Conflict of Interests}

The authors declare that there is no conflict of interests regarding the publication of this paper.

\section{Acknowledgments}

This paper was funded by King Abdulaziz University, under Grant no. 23-130-1433-HiCi. The authors, therefore, acknowledge the technical and financial support of KAU. The authors would like to thank the referees for their sincere evaluation and constructive comments which improved the paper considerably.

\section{References}

[1] S. Adly, "Perturbed algorithms and sensitivity analysis for a general class of variational inclusions," Journal of Mathematical Analysis and Applications, vol. 201, no. 2, pp. 609-630, 1996.

[2] A. Alotaibi, V. Kumar, and N. Hussain, "Convergence comparison and stability of Jungck-Kirk type algorithms for common fixed point problems," Fixed Point Theory and Applications, vol. 2013, article 173, 2013.

[3] L. C. Ceng, N. Husain, A. Latif, and J. C. Yao, "Strong convergence for solving general system of variational inequalities and fixed point problems in Banach spaces," Journal of Inequalities and Applications, vol. 2013, article 334, 2013.

[4] H. R. Feng and X. P. Ding, "A new system of generalized nonlinear quasi-variational-like inclusions with $A$-monotone operators in Banach spaces," Journal of Computational and Applied Mathematics, vol. 225, no. 2, pp. 365-373, 2009.

[5] H.-Y. Lan, Y. J. Cho, and R. U. Verma, "Nonlinear relaxed cocoercive variational inclusions involving $(A, \eta)$-accretive mappings in Banach spaces," Computers and Mathematics with Applications, vol. 51, no. 9-10, pp. 1529-1538, 2006.

[6] Y.-P. Fang and N.-J. Huang, " $H$-accretive operators and resolvent operator technique for solving variational inclusions in Banach spaces," Applied Mathematics Letters, vol. 17, no. 6, pp. 647-653, 2004.

[7] Z. B. Wang and X. P. Ding, " $H((.,),. \eta)$-accretive operators with an application for solving set-valued variational inclusions in Banach spaces," Computers and Mathematics with Applications, vol. 59, no. 4, pp. 1559-1567, 2010.

[8] Y.-Z. Zou and N.-J. Huang, "H(., .)-accretive operator with an application for solving variational inclusions in Banach spaces," Applied Mathematics and Computation, vol. 204, no. 2, pp. 809816, 2008.

[9] M.-M. Jin, "Convergence and stability of iterative algorithm for a new system of $(A, \eta)$-accretive mapping inclusions in Banach spaces," Computers and Mathematics with Applications, vol. 56, no. 9, pp. 2305-2311, 2008.

[10] H.-Y. Lan, "Stability of iterative processes with errors for a system of nonlinear $(A, \eta)$-accretive variational inclusions in Banach spaces," Computers and Mathematics with Applications, vol. 56, no. 1, pp. 290-303, 2008.

[11] X.-P. Luo and N.-J. Huang, " $(H, \phi)-\eta$-monotone operators in Banach spaces with an application to variational inclusions," Applied Mathematics and Computation, vol. 216, no. 4, pp. 11311139, 2010.

[12] Z. Xu and Z. Wang, "A Generalized mixed variational inclusion involving $(H(.,),. \eta)$-monotone operators in Banach spaces," Journal of Mathematics Research, vol. 2, no. 3, pp. 47-56, 2010.

[13] R. U. Verma, "Approximation solvability of a class of nonlinear set-valued variational inclusions involving $(A, \eta)$-monotone mappings," Journal of Mathematical Analysis and Applications, vol. 337, no. 2, pp. 969-975, 2008.

[14] H.-Y. Lan, "Nonlinear parametric multi-valued variational inclusion systems involving $(A, \eta)$-accretive mappings in Banach spaces," Nonlinear Analysis: Theory, Methods of Applications, vol. 69, no. 5-6, pp. 1757-1767, 2008.

[15] H.-Y. Lan, " $(A, \eta)$-accretive mappings and set-valued variational inclusions with relaxed cocoercive mappings in Banach spaces," Applied Mathematics Letters, vol. 20, no. 5, pp. 571-577, 2007. 
[16] C.-L. Su, "Finger shape expansion and recognition by wavelet transform," Applied Mathematics and Computation, vol. 190, no. 2, pp. 1583-1592, 2007.

[17] H.-Y. Lan, Y.-S. Cui, and Y. Fu, "New approximation-solvability of general nonlinear operator inclusion couples involving $(A, \eta, m)$-resolvent operators and relaxed cocoercive type operators," Communications in Nonlinear Science and Numerical Simulation, vol. 17, no. 4, pp. 1844-1851, 2012.

[18] N. Hussain and A. Rafiq, "On modified implicit Mann iteration method involving strictly hemicontractive mappings in smooth Banach spaces," Journal of Computational Analysis and Applications, vol. 15, no. 5, pp. 892-902, 2013.

[19] N. Hussain, A. Rafiq, and L. B. Ciric, "Stability of the Ishikawa iteration scheme with errors for two strictly hemicontractive operators in Banach spaces," Fixed Point Theory and Applications, vol. 2012, article 160, 2012.

[20] S. H. Khan, A. Rafiq, and N. Hussain, "A three-step iterative scheme for solving nonlinear $\phi$-strongly accretive operator equations in Banach spaces," Fixed Point Theory and Applications, vol. 2012, article 149, 2012.

[21] L.-C. Ceng, A. R. Khan, Q. H. Ansari, and J.-C. Yao, "Viscosity approximation methods for strongly positive and monotone operators," Fixed Point Theory, vol. 10, no. 1, pp. 35-71, 2009.

[22] R. Ahmad and M. Mursaleen, "System of generalized $H$ resolvent equations and the corresponding system of generalized variational inclusions," Hacettepe Journal of Mathematics and Statistics, vol. 41, no. 1, pp. 33-45, 2012.

[23] R. Ahmad, M. Dilshad, M.-M. Wong, and J.-C. Yao, "Generalized set-valued variational-like inclusions involving $H(.,)-.\eta$ cocoercive operator in Banach spaces," Journal of Inequalities and Applications, vol. 2012, article 149, 2012.

[24] S. B. Nadler, "Multivalued contraction mappings," Pacific Journal of Mathematics, vol. 30, no. 3, pp. 457-488, 1969.

[25] I. Cioranescu, Geometry of Banach Spaces, Duality Mappings and Nonlinear Problems, vol. 62 of Mathematics and Its Applications, Kluwer Academic Publishers, Dordrecht, The Netherlands, 1990.

[26] W. V. Petryshyn, "A characterization of strict convexity of Banach spaces and other uses of duality mappings," Journal of Functional Analysis, vol. 6, no. 2, pp. 282-291, 1970.

[27] R. Ahmad, M. Akram, and J. C. Yao, "Generalized monotone mapping with an application for solving a variational inclusion problem," Journal of Optimization Theroy and Applications, vol. 157, no. 2, pp. 324-346, 2013. 


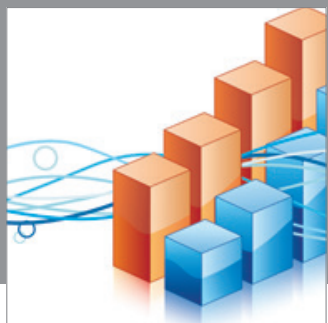

Advances in

Operations Research

mansans

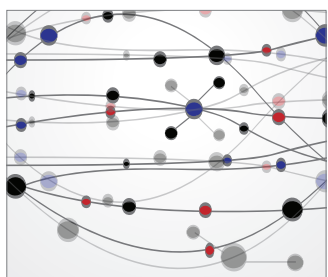

The Scientific World Journal
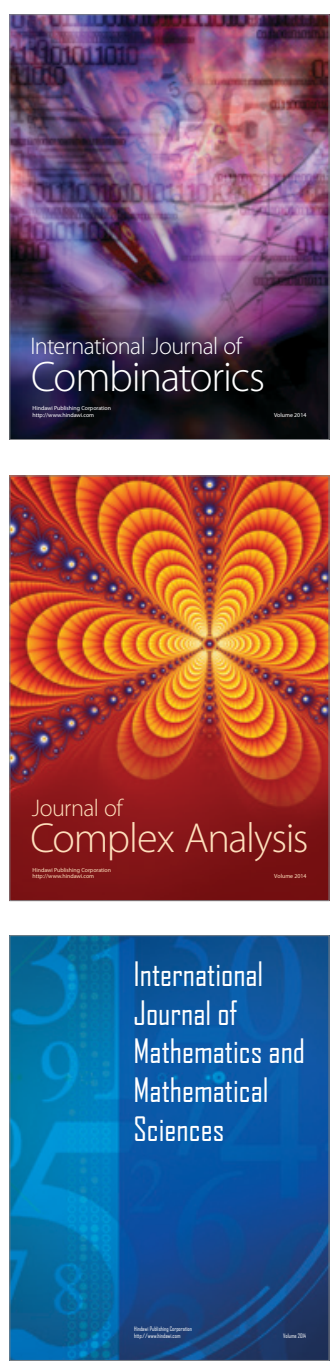
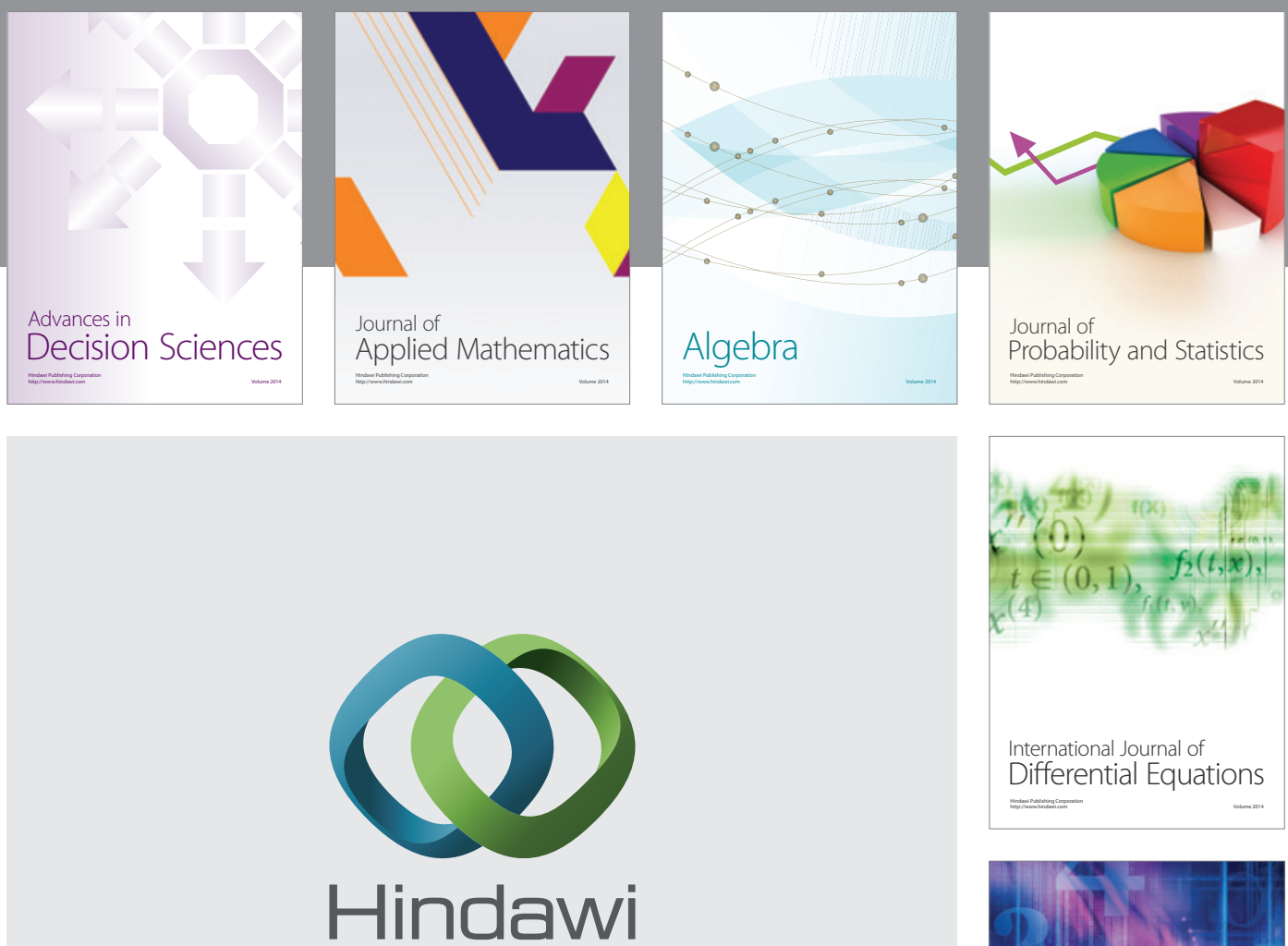

Submit your manuscripts at http://www.hindawi.com
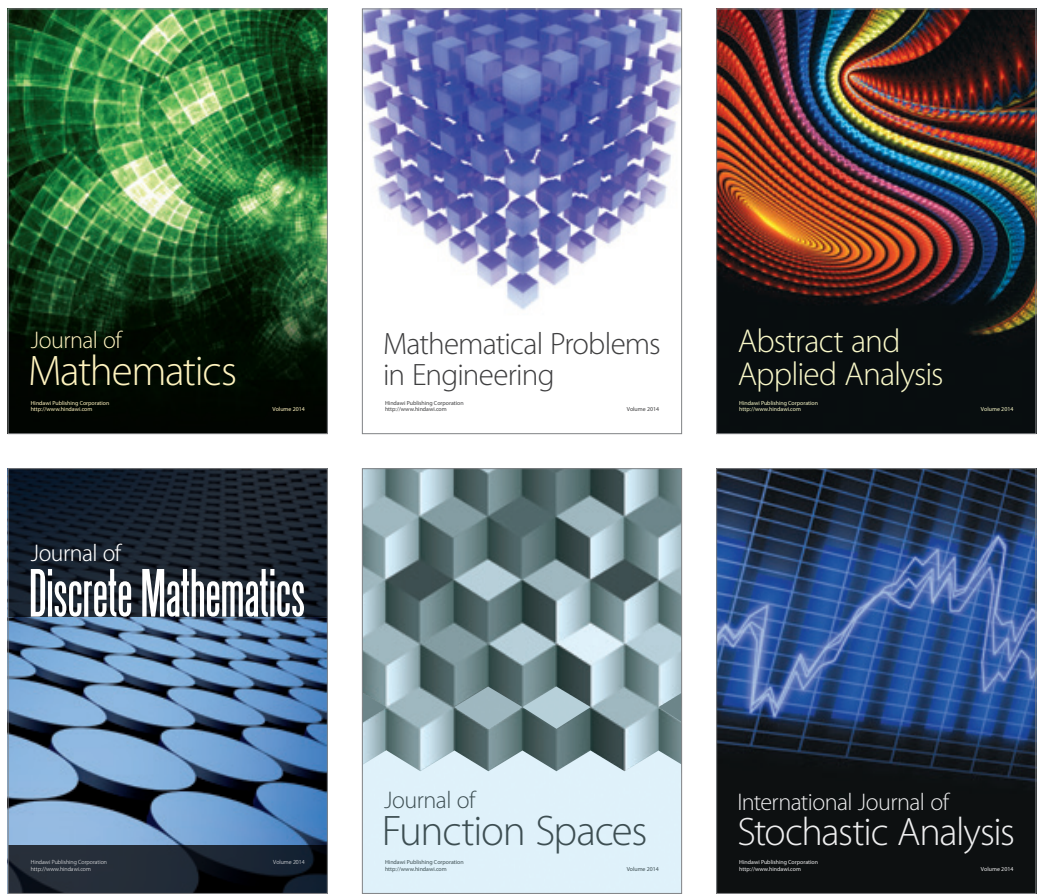

Journal of

Function Spaces

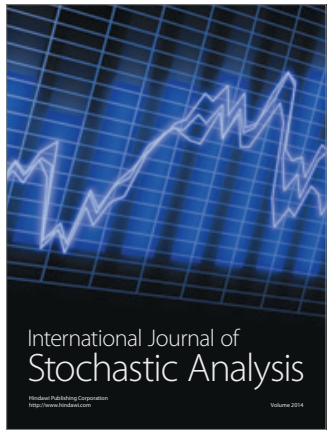

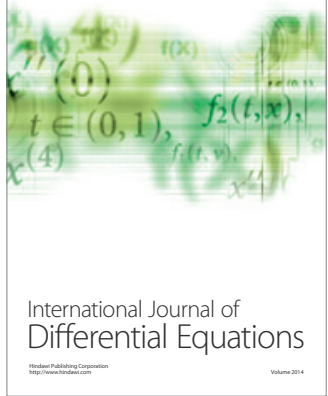
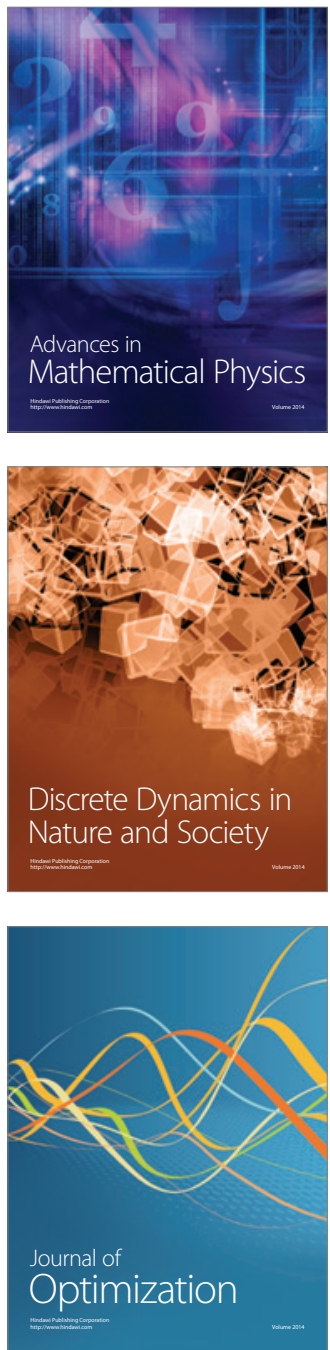